\title{
Global phosphoproteomics reveals DYRK1A regulates CDK1 activity in glioblastoma cells
}

\author{
Ariadna Recasens', Sean J. Humphrey $\mathbb{D}^{2}$, Michael Ellis ${ }^{1}$, Monira Hoque ${ }^{1}$, Ramzi H. Abbassi ${ }^{1}$, Brianna Chen', \\ Mitchell Longworth ${ }^{3}$, Elise J. Needham², David E. James $\mathbb{D}^{2}$, Terrance G. Johns ${ }^{4}$, Bryan W. Day ${ }^{5}$, Michael Kassiou $\mathbb{0}^{3}$, \\ Pengyi Yang $\mathbb{1}^{1,6,7}$ and Lenka Munoz (1)
}

\begin{abstract}
Both tumour suppressive and oncogenic functions have been reported for dual-specificity tyrosine phosphorylationregulated kinase 1A (DYRK1A). Herein, we performed a detailed investigation to delineate the role of DYRK1A in glioblastoma. Our phosphoproteomic and mechanistic studies show that DYRK1A induces degradation of cyclin B by phosphorylating CDC23, which is necessary for the function of the anaphase-promoting complex, a ubiquitin ligase that degrades mitotic proteins. DYRK1A inhibition leads to the accumulation of cyclin B and activation of CDK1. Importantly, we established that the phenotypic response of glioblastoma cells to DYRK1A inhibition depends on both retinoblastoma (RB) expression and the degree of residual DYRK1A activity. Moderate DYRK1A inhibition leads to moderate cyclin B accumulation, CDK1 activation and increased proliferation in RB-deficient cells. In RB-proficient cells, cyclin B/CDK1 activation in response to DYRK1A inhibition is neutralized by the RB pathway, resulting in an unchanged proliferation rate. In contrast, complete DYRK1A inhibition with high doses of inhibitors results in massive cyclin B accumulation, saturation of CDK1 activity and cell cycle arrest, regardless of RB status. These findings provide new insights into the complexity of context-dependent DYRK1A signalling in cancer cells.
\end{abstract}

\section{Introduction}

Dual-specificity tyrosine phosphorylation-regulated kinase 1A (DYRK1A) is activated by autophosphorylation of tyrosine residues during protein translation. Thus, the mature DYRK1A protein is a constitutively active kinase whose signalling via phosphorylation of downstream substrates at serine/threonine residues is regulated largely by DYRK1A expression levels, known as the dosage effect ${ }^{1}$. DYRK1A has been implicated in a diverse variety of biological processes, including CNS development, Down syndrome, beta-cell proliferation and diabetes, as well as Alzheimer's disease ${ }^{1-6}$. However, the

\footnotetext{
Correspondence: Ariadna Recasens (ariadna.recasens@sydney.edu.au) or Lenka Munoz (lenka.munoz@sydney.edu.au)

${ }^{1}$ Charles Perkins Centre and School of Medical Sciences, Faculty of Medicine and Health, The University of Sydney, Camperdown, NSW 2006, Australia ${ }^{2}$ Charles Perkins Centre and School of Life and Environmental Sciences, Faculty of Science, The University of Sydney, Camperdown, NSW 2006, Australia Full list of author information is available at the end of the article Edited by D. Michod
}

role of DYRK1A in cancer is not fully understood, and both oncogenic and tumour suppressive roles for DYRK1A have been reported ${ }^{7,8}$. For example, DYRK1A functions as a tumour suppressor in breast cancer and acute myeloid leukaemia ${ }^{9,10}$. In contrast, some evidence suggests oncogenic roles for DYRK1A, via stabilization of receptor tyrosine kinases ${ }^{7,11}$ and acceleration of tumour growth $^{12,13}$. Further adding to the DYRK1A controversy, DYRK1A inhibitors have been repeatedly reported to exert anti-proliferative activity when tested on cancer cells $^{14-17}$. Off-target effects are well-known players in the anti-cancer efficacy of kinase inhibitors ${ }^{18}$ and kinome screens demonstrate that DYRK1A inhibitors potently inhibit other kinases ${ }^{3-6}$. Nevertheless, a recent study using six orthogonal DYRK1A inhibitors demonstrated that the off-target effects are redundant with respect to the proliferative effects of DYRK1A inhibitors, at least in pancreatic beta cells ${ }^{4}$.

\section{(c) The Author(s) 2021}

(c) (i) Open Access This article is licensed under a Creative Commons Attribution 4.0 International License, which permits use, sharing, adaptation, distribution and reproduction cc) in any medium or format, as long as you give appropriate credit to the original author(s) and the source, provide a link to the Creative Commons license, and indicate if changes were made. The images or other third party material in this article are included in the article's Creative Commons license, unless indicated otherwise in a credit line to the material. If material is not included in the article's Creative Commons license and your intended use is not permitted by statutory regulation or exceeds the permitted use, you will need to obtain permission directly from the copyright holder. To view a copy of this license, visit http://creativecommons.org/licenses/by/4.0/. 
Glioblastoma is a lethal brain tumour characterized by molecular heterogeneity and aberrant cellular hierarchy. At the apex of this hierarchy sits a small population of glioblastoma stem cells, which are key mediators of tumour propagation and therapy failure ${ }^{19}$. Inhibition of DYRK1A in glioblastoma stem cell induces degradation of epidermal growth factor receptor (EGFR), resulting in decreased selfrenewal of EGFR-addicted cells ${ }^{7}$. In contrast, evidence suggests that elevated expression of DYRK1A leads to destabilization of hypoxia-inducible factor $2 \alpha$, loss of glioblastoma stemness and inhibition of tumour growth ${ }^{8}$. Furthermore, DYRK1A-regulated DREAM assembly and quiescence were established in glioblastoma cells where DYRK1A was ectopically over-expressed ${ }^{20}$. Collectively, while these observations make it clear that DYRK1A is an important kinase in glioblastoma, they also suggest that the function of DYRK1A in glioblastoma, and probably in other tumours, is dependent on the cell type and its genetic background.

In this study, we delineate a novel DYRK1A signalling pathway in glioblastoma stem cells. Phosphoproteomic and mechanistic studies revealed that DYRK1A regulates the anaphase-promoting complex (APC) ligase complex and degradation of cyclin $\mathrm{B}$, the primary co-activator of the CDK1. We confirmed that DYRK1A inhibition leads to CDK1 activation. Importantly, we discovered that the proliferative response to CDK1 activation upon DYRK1A inhibition is determined by the retinoblastoma (RB) expression and the degree of residual DYRK1A activity.

\section{Results}

Phosphoproteomics reveals that DYRK1A regulates CDK1

To obtain a global view of DYRK1A-regulated pathways, we analysed the proteomes and phosphoproteomes of U251 glioblastoma cells infected with doxycyclineinducible DYRK1A shRNA (Fig. 1A) or treated with DYRK1A inhibitors leucettine $41\left(\mathrm{~L} 41, \mathrm{IC}_{50}=40 \mathrm{nM}\right)^{21}$ and ALGERNON (ALG, $\left.\quad \mathrm{IC}_{50}=77 \mathrm{nM}\right)^{22}$. Target engagement was confirmed by decreased phosphorylation of cyclin D1 (Fig. 1B), a direct DYRK1A downstream substrate $^{23}$. Single-run proteome measurements identified $\sim 7400$ proteins (Fig. 1C and Table S1) with high reproducibility (Pearson correlation $>0.98$ for all samples; Fig. S1A). Globally, DYRK1A inhibitors changed expression levels of 1200 proteins, while knockdown of DYRK1A changed levels of 220 proteins (Figs. 1D and S1B). Around $50 \%$ of the proteins affected by DYRK1A knockdown were also affected by inhibitors, while only $10 \%$ of those affected by inhibitors were also affected by knockdown (Fig. 1D). This suggests that DYRK1A inhibitors likely exhibit some off-target effects.

To quantify changes to signalling of glioblastoma cells induced by DYRK1A inhibition, we employed the EasyPhos phosphoproteomics workflow ${ }^{24,25}$. In untreated cells, we quantified over 28,000 phosphorylation sites located on 4499 proteins (Fig. 2A). Globally, 84\% of phosphorylation sites were localized to a single amino acid with high precision (median localization probability 0.99; Table S2). Quantification coverage was high, with over 15,000 phosphorylation sites quantified in each sample with high reproducibility (Figs. 2A and S2A).

As with the proteome, DYRK1A inhibitors exerted more pronounced effects on the phosphoproteome than did DYRK1A knockdown (Fig. S2B), and we observed similar overlap in regulated phosphorylation events in response to each treatment type (Fig. 2B). While phosphorylation sites that are unique to pharmacological inhibition may include off-target effects of these inhibitors, sites overlapping with DYRK1A knockdown are likely enriched in bona fide DYRK1A substrates. To explore phosphorylation sites regulated by both pharmacological and genetic DYRK1A inhibition, we performed an analysis of the amino acid sequences surrounding phosphorylated residue, revealing a strong preference for Proline $(\mathrm{P})$ in the +1 position (Fig. $\mathrm{S} 2 \mathrm{C}$ ). DYRK1A is a basophilic kinase ${ }^{26}$, and in agreement with this, we observed an enrichment of Arginine (R) in the -3 position of these phosphorylation sites. Taking this as a 'minimal consensus sequence' filter and applying it to the 950 commonly regulated sites highlighted 61 phosphorylation sites that may represent putative DYRK1A substrates (Fig. $2 \mathrm{C}, \mathrm{D})$. Of these 61 phosphorylation sites, almost all were decreased in several treatment conditions compared to control samples (Fig. 2D) and were not decreased at the protein level (Fig. 2E), strengthening our hypothesis that these may be enriched in direct DYRK1A substrates.

Analysis of sites hyperphosphorylated upon $10 \mu \mathrm{M}$ ALG and DYRK1A knockdown revealed an enrichment of CDK and RAF1 kinase-substrate motifs (Fig. 2F, G). On a pathway level, we observed enrichment of cell cycle, DNA replication and mitotic processes (Fig. 2H). Given the robust substrate motif enrichment for CDK1/2 (Fig. 2F), we investigated phosphorylation changes in CDK1 substrates in more detail. From a total of 534 reported CDK1 substrates (www.phosphosite.org), 148 were present in our phosphoproteomic dataset. Of these, 115 (77\%) displayed increased phosphorylation after DYRK1A inhibition, with 37 of these being significantly hyperphosphorylated (Fig. 2I and Table S3). These analyses suggest for the first time that DYRK1A inhibition leads to CDK1 activation.

\section{DYRK1A regulates cyclin B degradation}

CDK1 activity is controlled by precise regulation of cyclin $B$ levels and CDK1 phosphorylation. Phosphorylation of CDK1 on residue T161 induces the conformational changes necessary for CDK1 activity, while T14 and Y15 phosphorylation inactivate CDK1 ${ }^{27}$. Our MS data revealed $\sim 2$ fold increased cyclin B levels, as well as hyperphosphorylation of CDK1 on Y15 and T161 in response to 


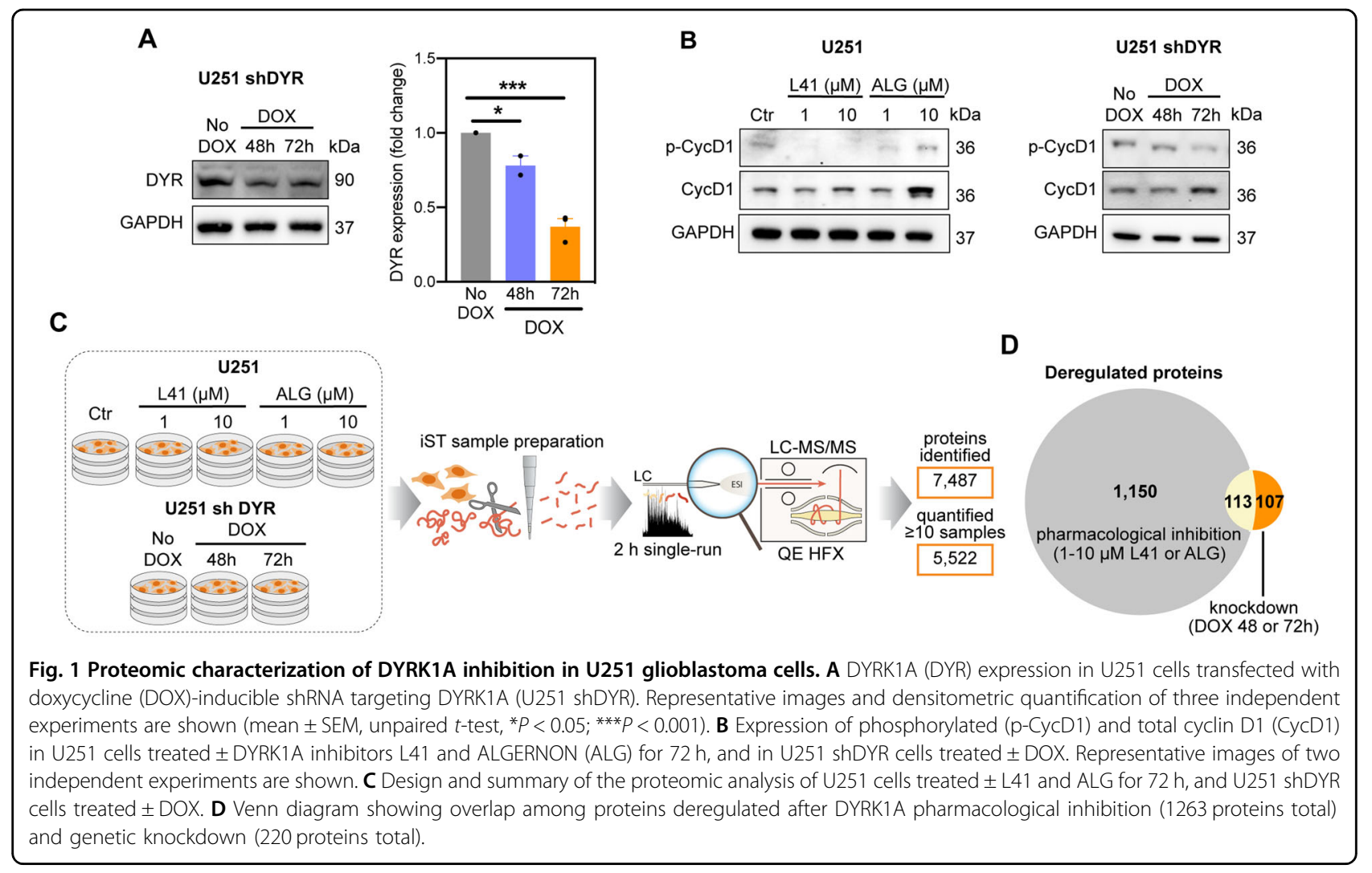

DYRK1A inhibition (Tables S1 and S2), which was confirmed by immunoblotting (Fig. 3A, B). Phosphorylation of the inhibitory $\mathrm{Y} 15$, despite the fact that CDK1 activity increased upon DYRK1A inhibition (Fig. 2F, G), is consistent with previous work showing that substantial CDK1 activity can occur concomitantly with high levels of CDK1 Y15 phosphorylation ${ }^{27,28}$. We also observed elevated, but not statistically significant CDK1 phosphorylation at the activating T161 upon DYRK1A inhibition. Nevertheless, our MS data did not reveal any changes in the expression or activity of kinases known to phosphorylate CDK1 at Y15 (Wee1, Myt1) and at T161 (CAK1) ${ }^{29}$ (Tables S1 and S2). Thus, given that CDK1 phosphorylation did not justify increased CDK1 activity, we concluded that DYRK1A inhibition likely leads to CDK1 activation by increasing cyclin B levels. In support, DYRK1A inhibition prevented degradation of cyclin B in U251 cells (Fig. 3C, D).

Cyclin B is primarily degraded by the APC composed of 11-13 proteins ${ }^{30}$. Phosphoproteomic analysis identified the APC subunit CDC23 as one of novel DYRK1A substrates, with CDC23 phosphorylation at S588 decreasing upon DYRK1A inhibition (Fig. 2D, asterix). In vitro kinase assays using recombinant proteins confirmed phosphorylation of CDC23 at S588 by DYRK1A (Figs. 3E and S3). Given that CDC23 phosphorylation is crucial for APC formation $^{31}$, we propose that decreased CDC23 phosphorylation after DYRK1A inhibition impairs proper functioning of the APC. Indeed, CDC23 knockdown increased the levels of cyclin B (Fig. 3F), and levels of additional APC substrates were increased in the proteome of U251 cells upon DYRK1A inhibition (Fig. 3G).

\section{DYRK1A regulates cell proliferation in a dose-dependent manner}

Since the CDK1/cyclin B complex is the master regulator of mitosis and pathway analysis indicates activation of mitosis upon DYRK1A inhibition (Fig. 2H), we next investigated the effect of DYRK1A inhibition on U251 cell proliferation. DYRK1A knockdown significantly increased cell numbers over 7 days (Fig. 4A). Similarly, the DYRK1A inhibitor ALG $(1 \mu \mathrm{M})$ increased the number of Ki67-positive cells and mitotic figures (Fig. 4B, C), especially during late mitotic telophase (Fig. $4 \mathrm{D})$, resulting in increased cell numbers and colony formation (Fig. 4E, F). Intriguingly, although $10 \mu \mathrm{M}$ ALG increased cyclin $B$ levels to a greater extent than the $1 \mu \mathrm{M}$ treatment (Fig. 3B), we did not observe a further increase in mitosis or proliferation (Fig. 4B-F). Microscopic images revealed the presence of morphologically normal cells, suggesting a cytostatic effect of ALG at $10 \mu \mathrm{M}$ (Fig. 4F). Similar findings were obtained using the orthogonal DYRK1A inhibitor L41 (Fig. 4F). Given that saturation of CDK1 activity achieved with nondegradable cyclin $\mathrm{B}$ leads to mitotic arrest ${ }^{32,33}$, these 


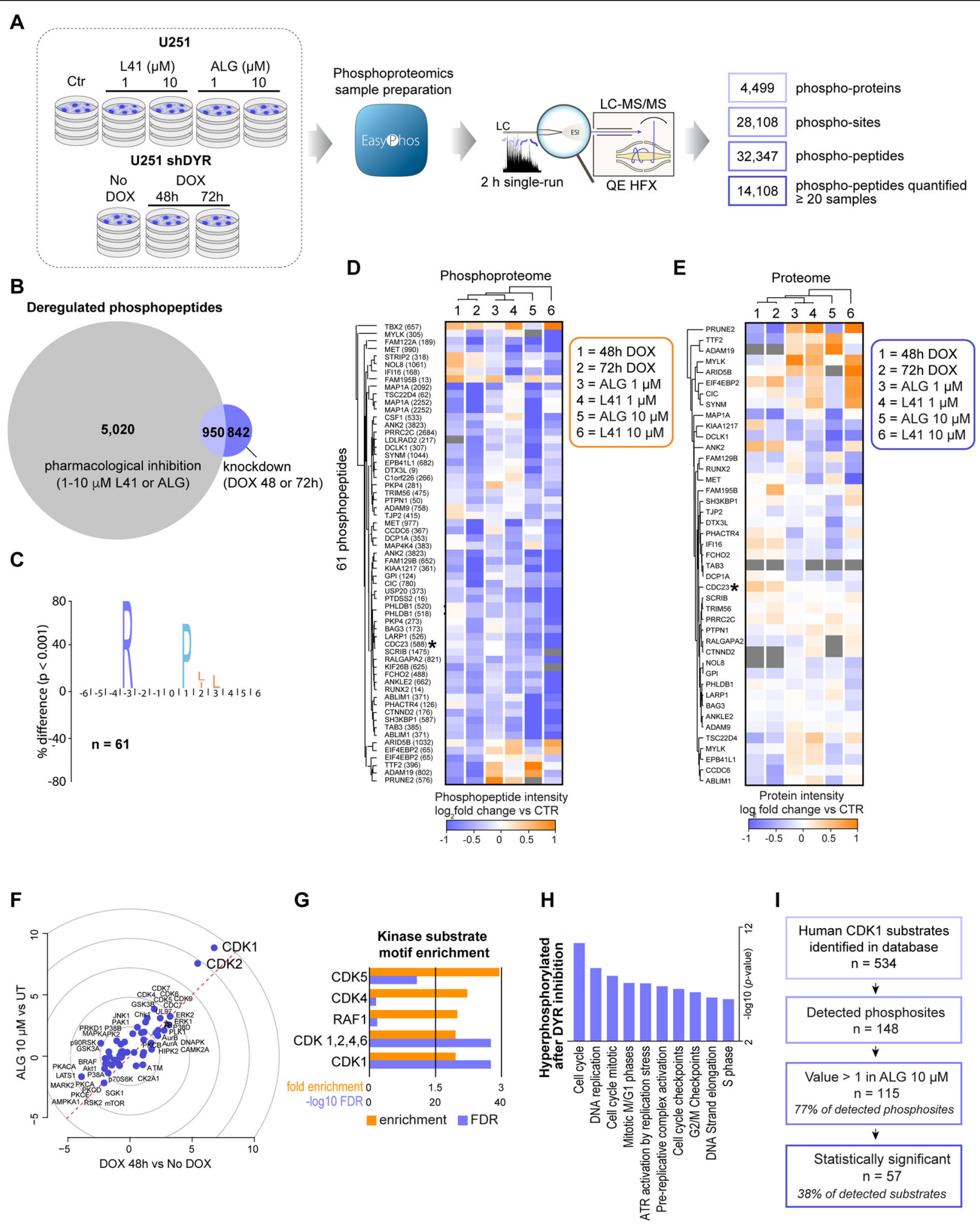

data suggest that complete DYRK1A inhibition results in cell cycle arrest due to immense accumulation of cyclin $\mathrm{B}$ and potentially, other mitotic regulators (Fig. 3G).
In support of the DYRK1A-CDC23 axis controlling glioblastoma cell cycle, CDC23 knockdown increased the percentage of Ki67-positive cells, the number of 
(see figure on previous page)

Fig. 2 Phosphoproteomic characterization of DYRK1A inhibition in U251 glioblastoma cells reveals CDK1 hyperactivity. A Design and summary of the phosphoproteomic analysis of U251 cells treated \pm DYRK1A inhibitors L41 and ALGERNON (ALG) for 72 h, and U251 shDYR cells treated \pm DOX. B Venn diagram showing overlap among phosphopeptides significantly (ANOVA adj. $P<0.05$ and Dunnett's $P<0.05$ ) reregulated by DYRK1A pharmacological inhibition (5,970 total) and genetic knockdown (1792 total). C A consensus sequence preference for DYRK1A. Motif enrichment analysis of 61 phosphopeptides regulated by both pharmacological and genetic inhibition that also possess an Arginine (R) in the -3 position, reveals a strong preference for Proline $(P)$ in the +1 position. D Heatmap of phosphopeptide intensities (median log2 fold changes for treatment vs their respective controls) for phosphopeptides identified as potential DYRK1A substrates. E Heatmap of protein changes (median log2 fold changes of protein LFQ intensities for treatments vs their respective controls) for proteins on which phosphosites from panel $D$ are found. F Kinase perturbation analysis with Kinase PA showing relative kinase activity in z-scores from DOX $48 \mathrm{~h}$ vs No DOX treatments ( $x$-axis) and ALG $(10 \mu \mathrm{M})$ vs untreated treatments (y-axis). G Kinase-substrate motif enrichment analysis for sites hyperphosphorylated in response to DYRK1A (DYR) inhibition. $\mathbf{H}$ Gene ontology over-representation analysis (Fischer's exact test) of significantly hyperphosphorylated proteins in cells treated with ALG $(10 \mu \mathrm{M}, 72 \mathrm{~h})$. I Annotation of CDK1 substrates (derived from PhosphoSitePlus) in the phosphoproteome of cells treated with ALG (10 $\mu \mathrm{M}, 72 \mathrm{~h}$ ).

mitotic figures and overall cell numbers (Fig. 4G-I). Thus, CDC23 knockdown phenocopied the effects of DYRK1A knockdown and low-dose ALG (Fig. 4A-F). Taken together, the proliferative response to DYRK1A inhibition, cyclin B accumulation and consequent CDK1 activation is dosedependent (Fig. 4). While partial DYRK1A inhibition by knockdown (protein levels reduced by $\sim 60 \%$ ) and lowdose inhibitors increased proliferation, complete DYRK1A inhibition with high-dose inhibitors results in cell cycle arrest.

\section{DYRK1A inhibition regulates proliferation in a cell- \\ dependent manner}

To investigate DYRK1A-CDK1 pathway in clinically relevant models, we knocked down DYRK1A in four patient-derived glioblastoma stem cell lines (Fig. 5A) ${ }^{34}$. Surprisingly, DYRK1A knockdown only increased proliferation in MMK1 cells, leaving the proliferation rates of HW1, RN1 and RKI1 cells unchanged (Fig. 5B). Further assays confirmed that DYRK1A knockdown increased MMK1 proliferation, whereas the proliferation rate of HW1 cells remained unchanged (Fig. 5C, D). Silencing DYRK1A increased the expression of S-phase (ORC1, MCM5) and mitotic (NEK2, BUB1) genes in both HW1 and MMK1 cell lines (Fig. 5E). Mitotic genes were upregulated to a greater extent in MMK1 cells, where we also observed increased proliferation (Fig. 5B-D). Flow cytometry revealed that DYRK1A knockdown increased accumulation of HW1 cells in S-phase (from basal 15\% to $21.4 \%$ ), whereas MMK1 cells accumulated in $\mathrm{G}_{2} / \mathrm{M}$ phase (from basal $4.8 \%$ to $7.1 \%$; Fig. 5F). Consistent with DYRK1A knockdown data, low-dose ALG $(1 \mu \mathrm{M})$ increased cell numbers, the number of mitotic figures and colony growth in MMK1 but not in HW1 cells (Fig. $5 \mathrm{G}-\mathrm{I})$. High-dose ALG $(10 \mu \mathrm{M})$ failed to further increase proliferation in MMK1 cells and showed cytostatic effects (Fig. 5H), in agreement with the results obtained in U251 cells. Thus, partial DYRK1A inhibition increased proliferation only in MMK1 cells, implying that the molecular profiles of cells shape the proliferative response to DYRK1A inhibition.

\section{Retinoblastoma expression determines the proliferative response to DYRK1A inhibition}

To further investigate different proliferation responses to DYRK1A inhibition, we first analysed the DYRK1A-cyclin B axis. DYRK1A silencing increased cyclin B levels in both MMK1 and HW1 cells (Fig. 6A, B), suggesting that additional mechanism controls the proliferative response to DYRK1A inhibition. We next investigated assembly of the DREAM complex, through which DYRK1A regulates $G_{0}$ cell cycle arrest (Fig. 6C) ${ }^{20}$. RNA sequencing ${ }^{34}$ and immunoblotting revealed that expression of DREAM proteins (low in HW1, RN1; high in MMK1, RKI1 cells; Fig. 6D, E) did not correlate with increased proliferation rates in MMK1 cells. Furthermore, we found that the DREAM complex is not assembled in glioblastoma stem cells, and knockdown of the key DREAM component P130 had no effect on MMK1 cell proliferation (Fig. 6F, G). DREAM assembly in A172 glioblastoma cells (Fig. 6F) served as a positive control for immunoprecipitation experiments ${ }^{35}$.

Next, we investigated compatibility of the RB pathway. HW1 and RN1 cells carry homozygous $C D K N 2 A / B$ deletion, whereas MMK1 and RKI1 cells carry heterozygous deletion and intact $C D K N 2 A / B$, respectively (Fig. 5A). $C D K N 2 A$ encodes the CDK inhibitor p16 that inhibits CDK4/6-dependent RB phosphorylation (Fig. 6H). RNAseq revealed higher expression of RB pathway components in RN1 and RKI1 cells compared to HW1 and MMK1 cells (Fig. 6I). Analysis of protein levels confirmed the absence of p16 in HW1 and RN1, low p16 expression in MMK1 and high levels in RKI1 cells (Fig. 6J). Protein levels of RB and cyclin D1 mirrored their mRNA profiles and confirmed that MMK1 cells lack RB protein (Fig. 6I, J).

Considering that RB-deficient MMK1 cells increased their proliferation rate upon DYRK1A inhibition (Fig. 5B), we hypothesized that RB compensates for DYRK1A loss in cell cycle regulation. Thus, we performed single and double RB and DYRK1A knockdown in RB-proficient cells (Fig. 6K). While single knockdown of either RB or DYRK1A did not change the proliferation rates, double knockdown significantly increased cell proliferation (Fig. 6L). Furthermore, DYRK1A knockdown increased RB 
expression by 2-fold (Fig. 6K). In summary, DYRK1A knockdown increased the expression of cyclin $\mathrm{B}$ in glioblastoma stem cells, regardless of their molecular background. However, this cyclin B accumulation selectively increased the proliferation rate of RB-deficient cells. In RB-proficient cells, cyclin B accumulation in response to DYRK1A inhibition appears to be neutralized by increased $\mathrm{RB}$ expression, resulting in unchanged proliferation rate.

\section{DYRK1A inhibition reactivates the cell cycle in dormant RB- deficient cells}

Finally, we questioned whether DYRK1A inhibition reactivates the cell cycle in dormant cancer cells, which create a pool of drug-tolerant persister cells surviving anti-proliferative drugs ${ }^{36}$. To isolate dormant cells, RBdeficient MMK1 cells were treated for 14 days with high concentration (25x $\mathrm{EC}_{50}$ from 5-day viability assay) of the microtubule-targeting agent colchicine (Fig. 7A) ${ }^{37}$. Expression of DYRK1A and lack of RB in dormant cells remained unchanged, although DYRK1A appears to be post-translationally modified in dormant cells, which changes its molecular weight (Fig. 7B). Cell cycle genes were decreased and dormancy genes were increased in dormant cells (Fig. 7C, D). Furthermore, dormant cells resumed proliferation upon colchicine removal (i.e. drug holidays), demonstrating a reversible dormancy phenotype (Fig. S4). To study the effect of DYRK1A inhibition on cell dormancy, dormant MMK1 cells were treated with a proliferation-promoting low-dose ALG $(1 \mu \mathrm{M})$ for 7 days (Fig. 7E). Consistent with our data in parental MMK1 cells (Figs. 5 and 6), ALG increased the percentage of Ki67-positive cells and overall cell numbers (Fig. 7E, F). Seven days of drug holidays initiated re-expression of cell cycle genes (Fig. 7G, orange bars) necessary for cell cycle recovery (Fig. S4). This effect was significantly enhanced by addition of ALG (Fig. 7G, pink bars), accompanied with increased cyclin B expression (Fig. 7H).

Many chemotherapeutics, including the experimental drug colchicine used herein, predominantly kill actively cycling cells, while sparing dormant cells ${ }^{36}$. Our findings that DYRK1A inhibition forced cells to leave the dormant state prompted us to investigate whether DYRK1A inhibitors could improve the efficacy of anti-proliferative drugs. Indeed, co-treatment with colchicine and ALG $(1 \mu \mathrm{M})$ reduced the number of dormant MMK1 cells compared to colchicine treatment alone (Fig. 7I). Lastly, we addressed RB and DYRK1A cooperativity in dormant glioblastoma cells. Given that DYRK1A inhibition did not increase proliferation in RB-proficient HW1, RN1 or RKI1 cells (Fig. 5B), we expected and confirmed that ALG did not decrease the number of colchicine-surviving dormant cells (Fig. 7J). Importantly, RB knockdown in HW1 cells combined with colchicine + ALG significantly reduced the number of dormant cells (Fig. 7K). These observations further demonstrate the cooperativity between RB and DYRK1A in cell cycle regulation.

\section{Discussion}

In this study, we demonstrate for the first time that DYRK1A negatively regulates mitosis. Thus, our work adds DYRK1A to the small category of kinases that function as tumour suppressors, such as PKC and MKK $4^{38,39}$. Moreover, we identified 61 putative, novel DYRK1A downstream targets and validated CDC23 as a bona fide DYRK1A substrate. We report that partial DYRK1A inhibition increases proliferation and reactivates cell cycle of dormant RB-deficient glioblastoma cells, thereby improving efficacy of anti-proliferative drugs. Finally, we report that complete DYRK1A inhibition causes cell cycle arrest.

Mechanistically, we linked DYRK1A inhibition to the accumulation of cyclin B, which in turn activated CDK1, a key regulator of mitosis. We propose a model in which DYRK1A phosphorylates CDC23, an essential subunit for the proper function of the APC ligase ${ }^{31}$. CDC23, CDC27 and $\mathrm{CDC} 16$ form the scaffolding tetratricopeptide repeat subcomplex of the APC, which is necessary for substrate recognition ${ }^{30}$. Disrupting the formation of this subcomplex via targeting any of its subunits prevents recruitment and ubiquitination of cyclin $\mathrm{B}^{40}$. Similar to our results with DYRK1A and CDC23 knockdown leading to cyclin B accumulation, CDC27 inhibition also attenuated cyclin $\mathrm{B}$ degradation ${ }^{41}$. In addition to controlling APC ligase, DYRK1A regulates the E3 ligase RNF169 $9^{42,43}$ and Von Hippel-Lindau ligase ${ }^{8}$ and we identified ligases DTX3l and TRIM56 as putative DYRK1A targets (Fig. 2D), suggesting that DYRK1A is an upstream regulator of the proteasome.

Inhibition of DYRK1A causes activation of mitotic CDK1 regardless of the transcriptomic profile of the cell. However, a particularly striking observation was that this CDK1 activation specifically affects proliferation of RBdeficient cells and does not work in RB-expressing cells. On a systems level, this indicates that RB and DYRK1A cooperatively regulate the cell cycle. $R B$ functions as a break in early $G_{0}$ and $G_{1}$ phases ${ }^{44}$, whereas DYRK1A slows down mitosis via cyclin $B$ degradation (shown in this study). Given that single knockdown of $R B$ or DYRK1A did not impact the cell proliferation rates, whereas knockdown of both DYRK1A and RB enhanced proliferation, it appears that DYRK1A is redundant to the presence of $R B$ and vice versa. Thus, it is plausible that $R B$ status is responsible for the variable proliferation phenotypes of DYRK1A targeting reported within the same histological cancer types ${ }^{7,12,35}$.

In addition to $\mathrm{RB}$ status impacting the behaviour of DYRK1A-targeted cells, we also observed that the cellular response to DYRK1A inhibition is dose-dependent. While 


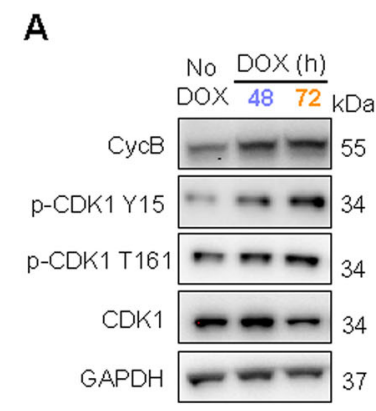

C

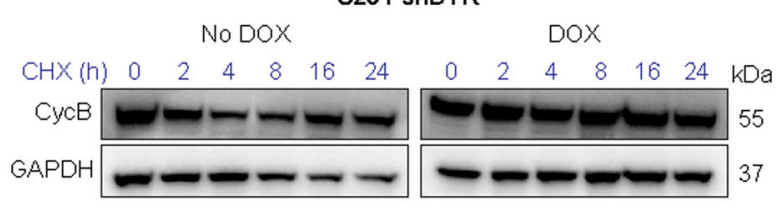

\section{B}

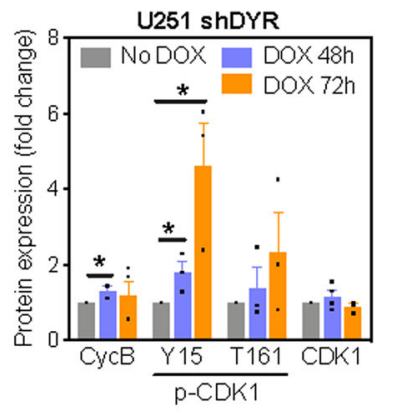

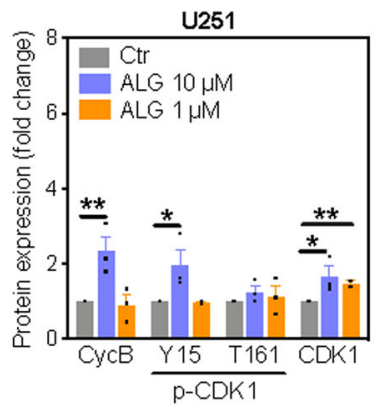

D

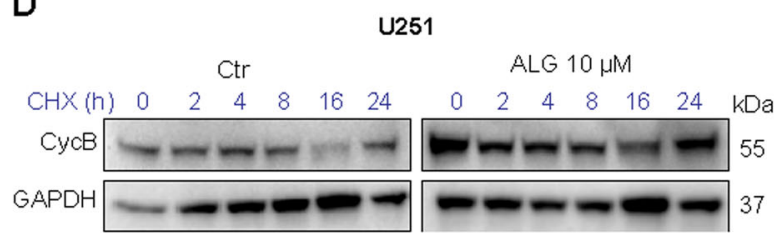

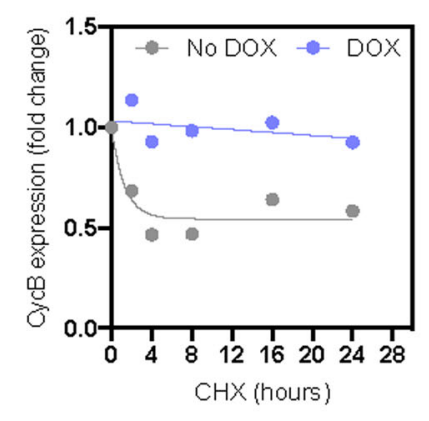

E

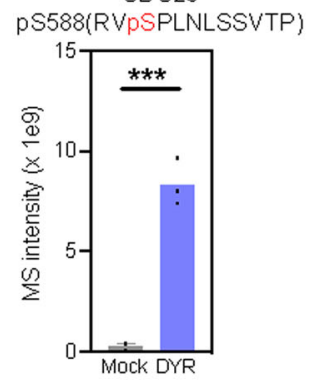

$\mathbf{F}$

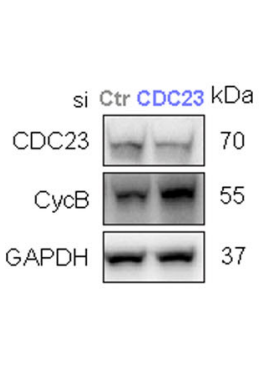

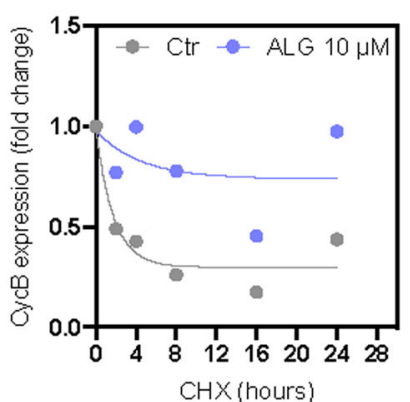

G

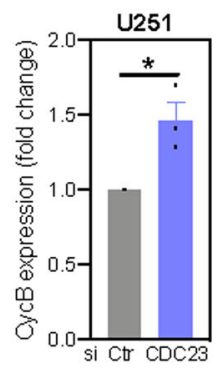

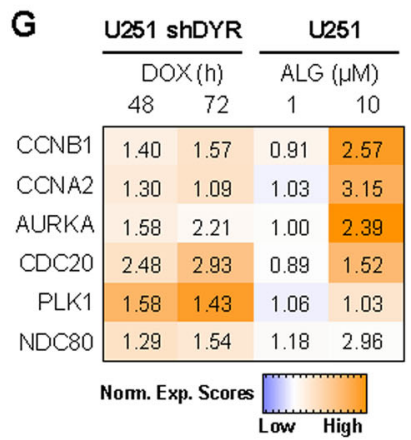

Fig. 3 DYRK1A inhibition prevents cyclin B degradation. Immunoblot analysis and densitometric quantification of cyclin B (CycB), phosphorylated ( $p$-CDK1) and total CDK1 in (A) U251 shDYR cells treated \pm DOX and (B) U251 cells treated \pm DYRK1A inhibitor ALGERNON (ALG, 72 h). Degradation of cyclin B (CycB) in U251 shDYR cells treated \pm DOX (C) and U251 cells treated \pm ALG (D). Cells were treated with DOX or ALG for $72 \mathrm{~h}$ before cycloheximide (CHX) treatment. Representative images and quantification from two independent experiments are shown. E Mass spectrometry (MS) intensity of CDC23 phosphorylated at S588 by DYRK1A in vitro. $\mathbf{F}$ Immunoblot analysis and densitometric quantification of cyclin B expression in cells transfected with siRNA targeting CDC23 (72 h). G Heatmap of protein levels of anaphase-promoting complex substrates cyclin B1 (CCNB1), cyclin A2 (CCNA2), Aurora kinase A (AURKA), cell-division cycle protein 20 (CDC20), Polo-like kinase 1 (PLK1) and kinetochore protein NDC80 in U251 cells following doxycycline-induced DYRK1A knockdown (DOX) and inhibition with ALG (72 h). Data represent fold change of replicates to their respective controls. All bar graphs represent mean \pm SEM of at least three independent experiments (two-tailed unpaired $t$-test; ${ }^{*} P<0.05$, $\left.{ }^{*} P<0.01,{ }^{* * *} P<0.001\right)$.

inhibition of DYRK1A systematically increased cyclin B levels in a dose-dependent fashion, only knockdown reducing DYRK1A levels by $\sim 60 \%$ and low-dose DYRK1A inhibitors increased proliferation. In contrast, DYRK1A inhibitors at high concentrations, despite further increasing cyclin $\mathrm{B}$ and CDK1 activity, had a cytostatic effect. 


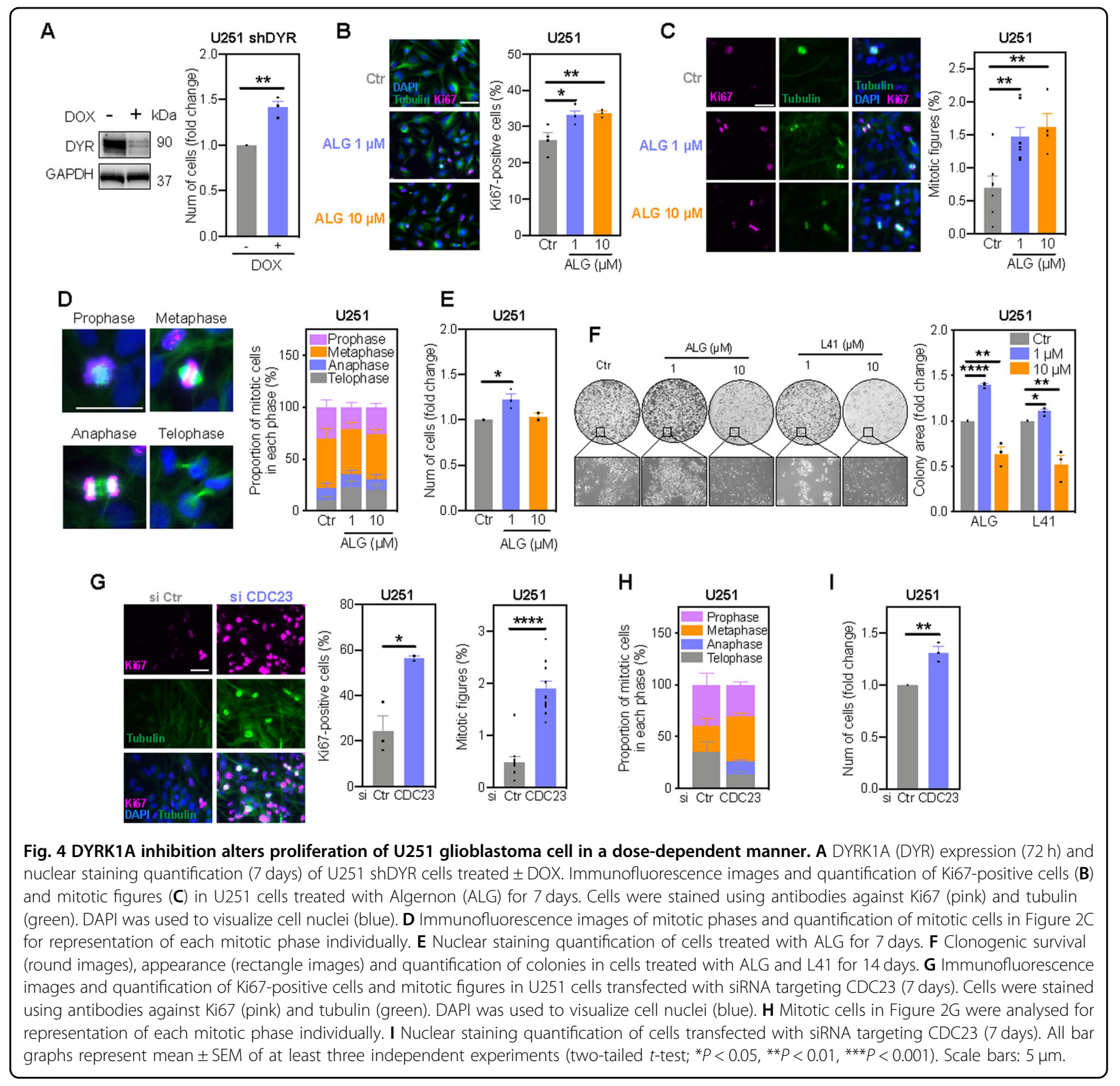

Consistent with our findings, dose-response curves for DYRK1A inhibitors revealed increasing rates of pancreatic beta-cell proliferation up to a given dose, after which proliferation declines ${ }^{4}$. Given that saturation of CDK1 activity with non-degradable cyclin $\mathrm{B}$ leads to mitotic arrest $^{32,33}$, we propose that complete DYRK1A inhibition with high concentrations of DYRK1A inhibitors and consequent massive accumulation of cyclin $\mathrm{B}$ also results in CDK1 saturation and cell cycle arrest (Fig. 7L). This conclusion explains the anti-proliferative effects of DYRK1A inhibitors reported by numerous studies, ${ }^{72,14-17,35}$. Inhibitors used in these studies inhibit DYRK1A with biochemical $\mathrm{IC}_{50}$ below $100 \mathrm{nM}$, and cellular target engagement has been demonstrated at $1 \mu \mathrm{M}^{14-17}$. Yet, in cell-based experiments, DYRK1A inhibitors were used at 10-30 $\mu \mathrm{M}$ concentration ${ }^{7,12,35}$. Finally, we show that low-dose DYRK1A inhibitors reactivate the cell cycle of dormant cells, thereby improving the efficacy of antiproliferative drugs. While DYRK1A inhibition enhanced the apoptotic efficacy of imatinib by disrupting DREAM complex in quiescent gastrointestinal cancer cells ${ }^{45}$, in glioblastoma cells, the anti-dormancy efficacy of DYRK1A inhibition is a result of CDK1 activation and is effective only in RB-deficient cells. 


\begin{tabular}{|c|c|c|c|c|c|}
\hline Pathway & Gene & HW1 & RN1 & MMK1 & RKI \\
\hline \multirow{2}{*}{ RTK } & EGFR & \multirow[t]{2}{*}{ A289V } & & & \\
\hline & MET & & T992I & & \\
\hline PI3K & PTEN & spl jnct & & F56V & \\
\hline MAPK & MYC & & & & \\
\hline RB & $\begin{array}{l}\text { CDKN2A } \\
\text { CDKN2B }\end{array}$ & & & & \\
\hline $\begin{array}{l}\text { hor } \\
\text { het }\end{array}$ & $\begin{array}{l}\text { zygous } \\
\text { ozygou }\end{array}$ & $\begin{array}{l}\text { deletion } \\
\text { deletion }\end{array}$ & & $\begin{array}{l}\text { amplif } \\
\text { gain }\end{array}$ & ation \\
\hline
\end{tabular}

C

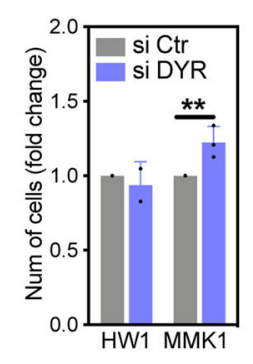

D

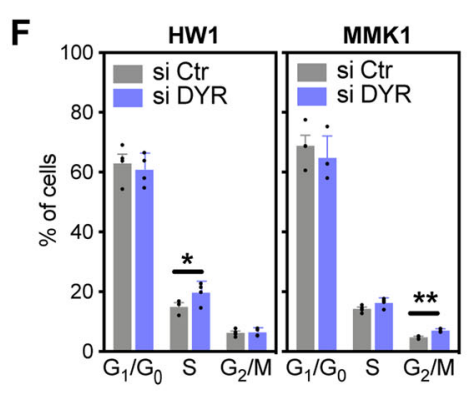

B

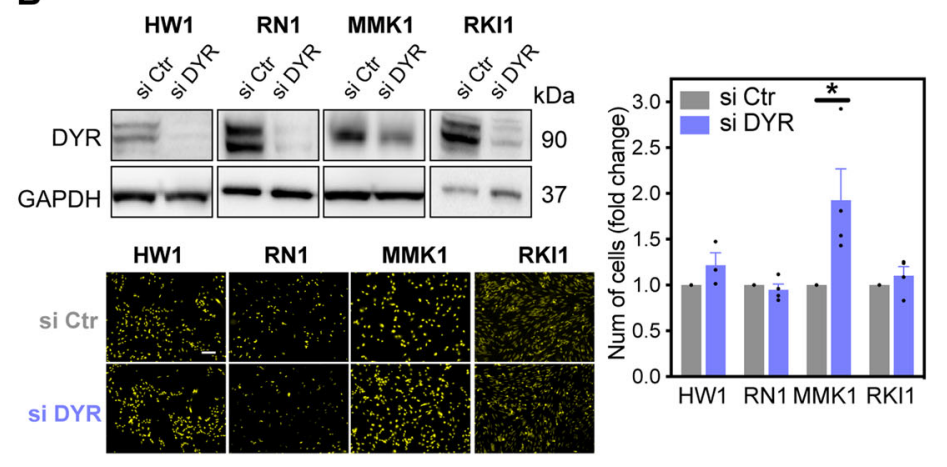

E

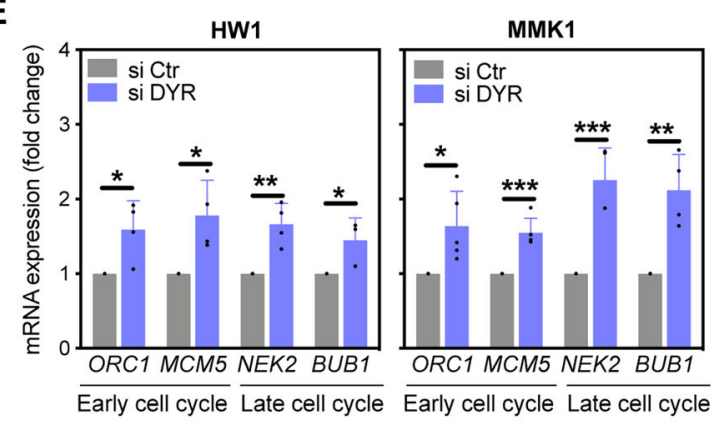

H

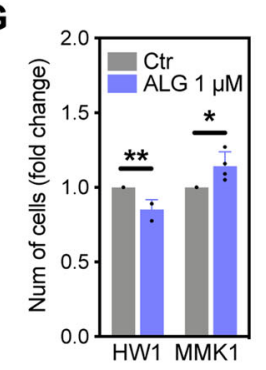

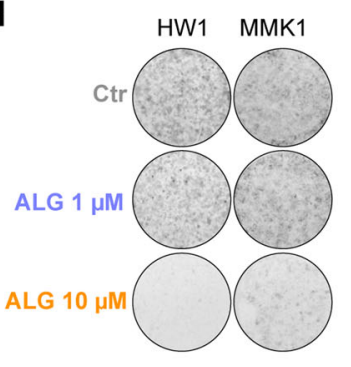

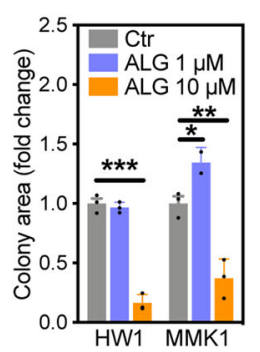

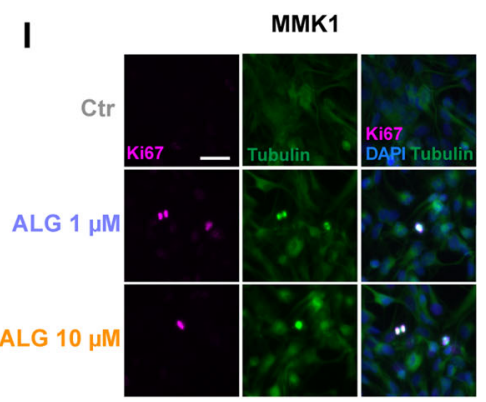
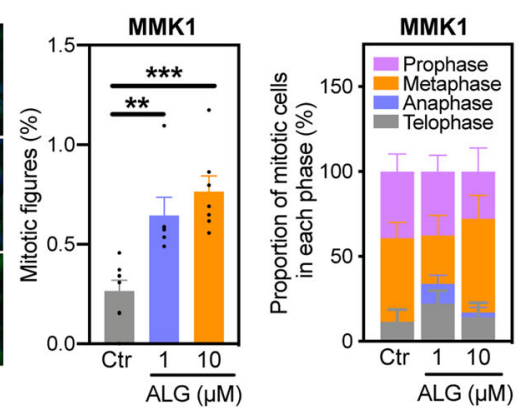

Fig. 5 DYRK1A inhibition regulates proliferation in a cell-dependent manner. A Genomic profiles of glioblastoma stem cell lines HW1, RN1, MMK1 and RKI. B Immunoblot analysis (72 h) and nuclear staining quantification (7 days) of cells transfected with scramble (si Ctr) and DYRK1Atargeting (si DYR) siRNAs. Scale bar: $10 \mu \mathrm{m}$. C Manual counting of cells transfected with si Ctr and si DYR for 7 days. D Representative images and quantification of colonies in cells transfected with si Ctr and si DYR for 14 days. E RT-PCR analysis of cell cycle genes in cells transfected with si Ctr and si DYR for 7 days. F Flow cytometry quantification of cells in $G 1 / G 0, S$ and $G_{2} / M$ phases following transfection with si Ctr and si DYR for 3 days. G Nuclear staining quantification of cells treated with DYRK1A inhibitor ALGERNON (ALG) for 7 days. H Representative images and quantification of colonies in cells treated with ALG for 14 days. I Immunofluorescence images and quantification of mitotic figures in cells treated with ALG for 7 days. Cells were stained using antibodies against Ki67 (pink) and tubulin (green). DAPI was used to visualize cell nuclei (blue). Scale bar: $5 \mu \mathrm{m}$. All bar graphs represent mean \pm SEM from at least three independent experiments (two-tailed, unpaired $t$-test, ${ }^{*} P<0.05,{ }^{* *} P<0.01,{ }^{* * *} P<0.001$ ). 
A

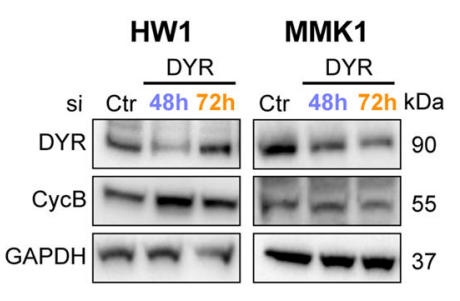

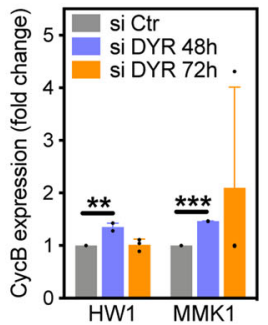

D

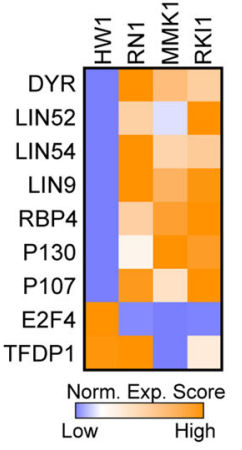

B
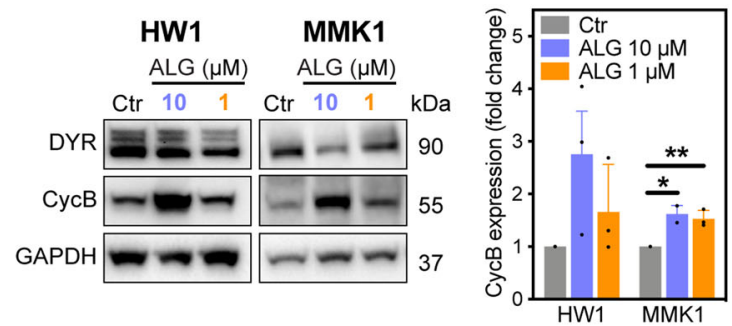

C

DREAM COMPLEX

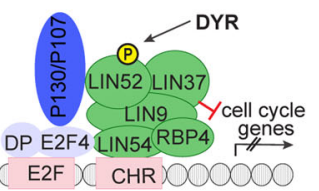

G

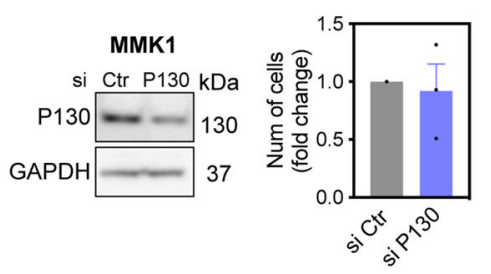

H
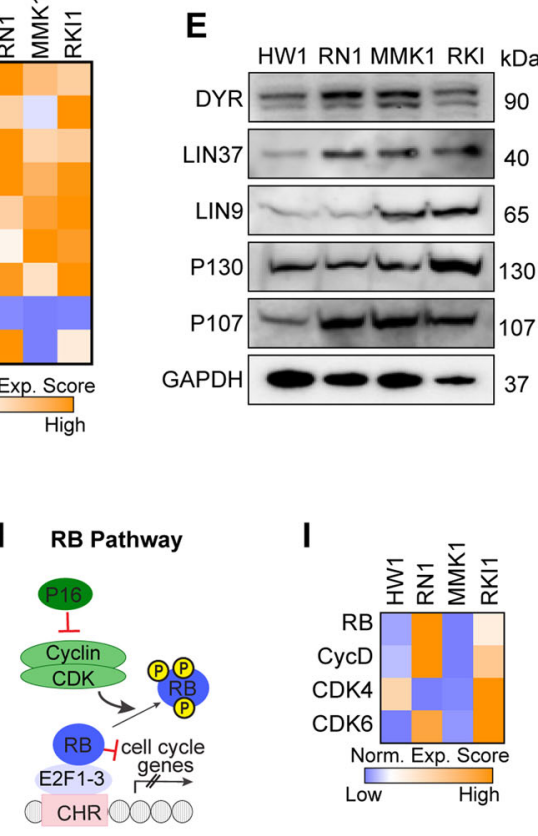

F

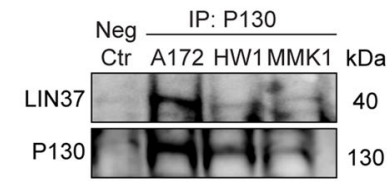

J

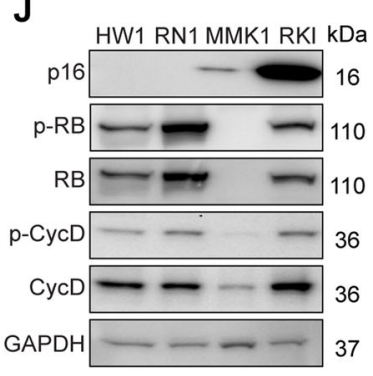

K

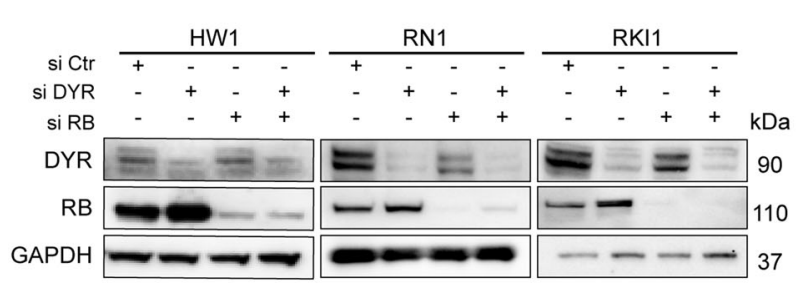

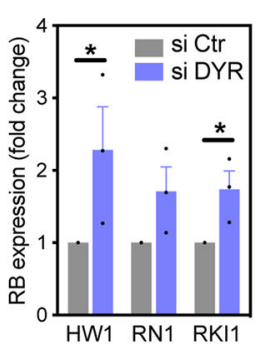

L

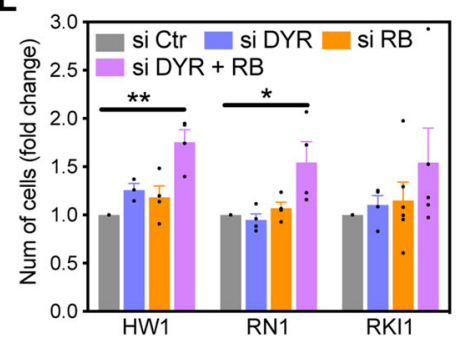

Fig. 6 Retinoblastoma expression determines the proliferative response to DYRK1A inhibition. A, B Immunoblot analysis and densitometric quantification of cyclin B (CycB) expression in cells treated with scramble (si Ctr) and DYRK1A (si DYR) targeting siRNA (72 h), and in cells treated with DYRK1A inhibitor ALGERNON (ALG, 72 h). C Schematic overview of DREAM complex. RNA seq data showing gene expression (D) and immunoblot analysis showing protein expression (E) of DREAM complex components in glioblastoma stem cells. $\mathbf{F}$ Immunoblot analysis of DREAM complex assembly in glioblastoma cells. Cell lysates were first immunoprecipitated (IP) with an anti-P130 antibody, then immunoblotted against P130 and LIN37. G Immunoblot analysis of MMK1 cells transfected with P130-targeting siRNA (si P130) for $72 \mathrm{~h}$ and nuclear staining quantification of cells transfected with si P130 for 7 days. $\mathbf{H}$ Schematic overview of retinoblastoma (RB) pathway. RNA seg data (I) and immunoblot analysis (J) of RB pathway components in glioblastoma stem cells. K, M Immunoblots analysis of cells transfected with scramble (si Ctr), DYRK1A-targeting (si DYR) and RB-targeting (si RB) siRNA for $72 \mathrm{~h}$. Densitometric quantification of RB expression following DYRK1A knockdown is shown. $\mathbf{L}$ Nuclear staining quantification of cells single transfected with si DYR or si RB, and double transfected (si DYR + RB) for 7 days. All bar graphs represent mean \pm SEM from at least three independent experiments (two-tailed unpaired t-test, ${ }^{*} P<0.05,{ }^{* *} P<0.01,{ }^{* * *} P<0.001$ ). 
A

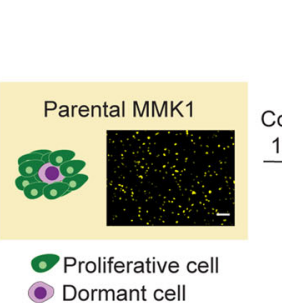

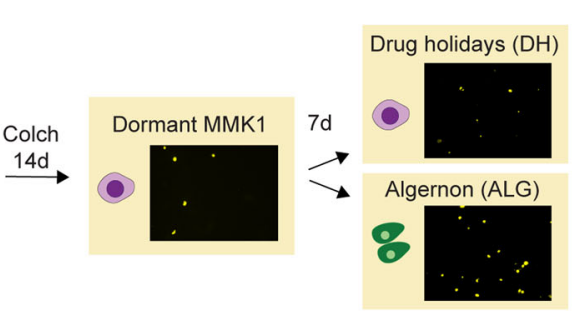

B

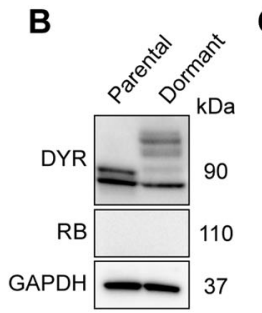

C

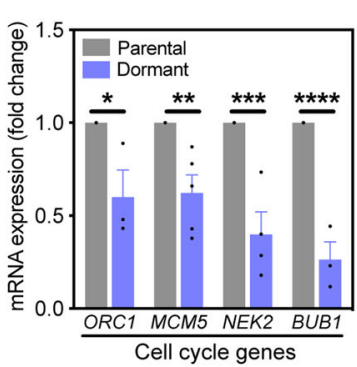

$\mathbf{F}$
D

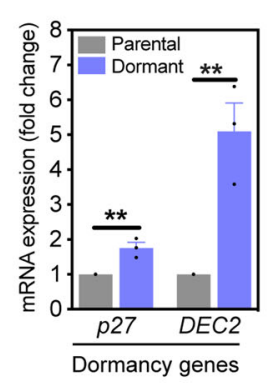

G

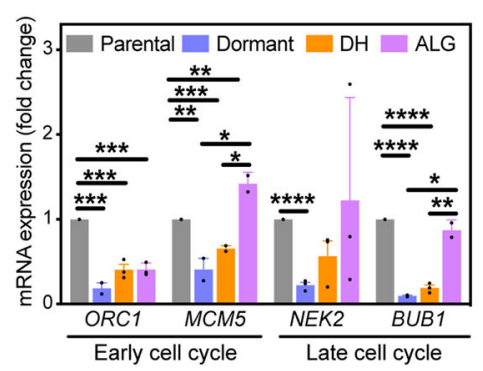

E

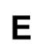

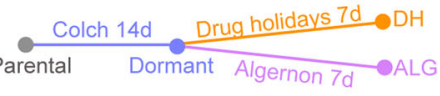

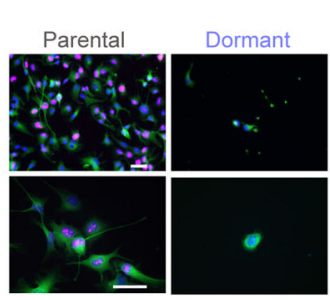

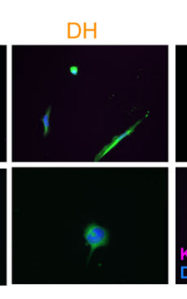
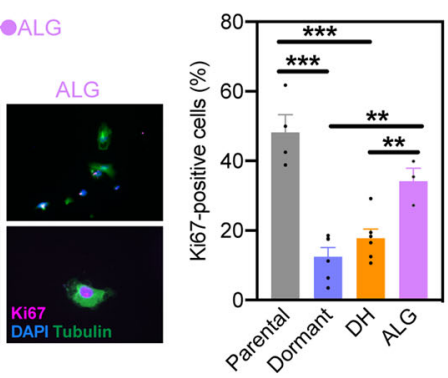

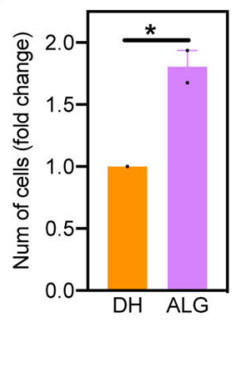

H

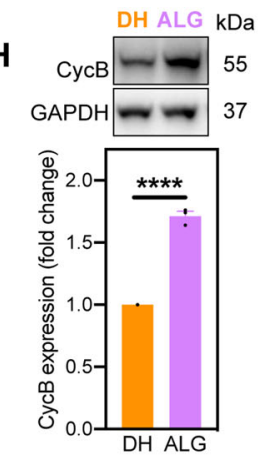

I MMk1
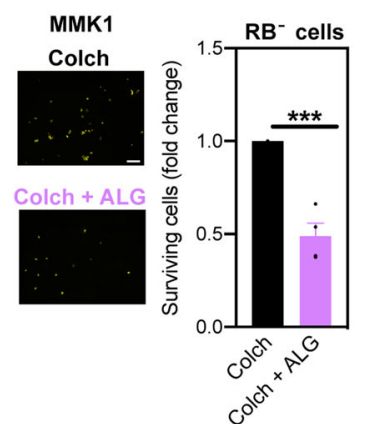

$\mathbf{J}$

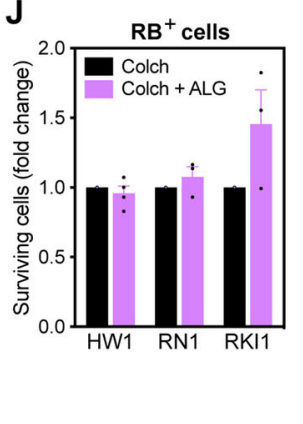

K
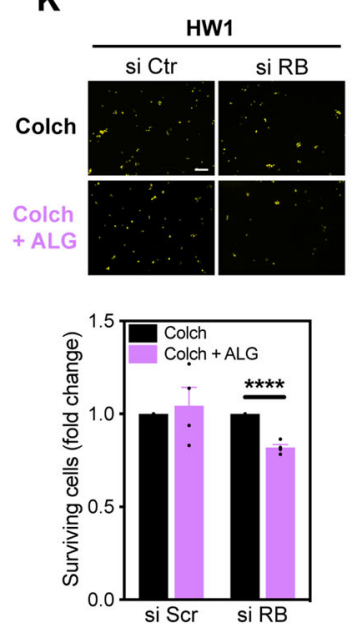

$\mathbf{L}$

DYRK1A activity

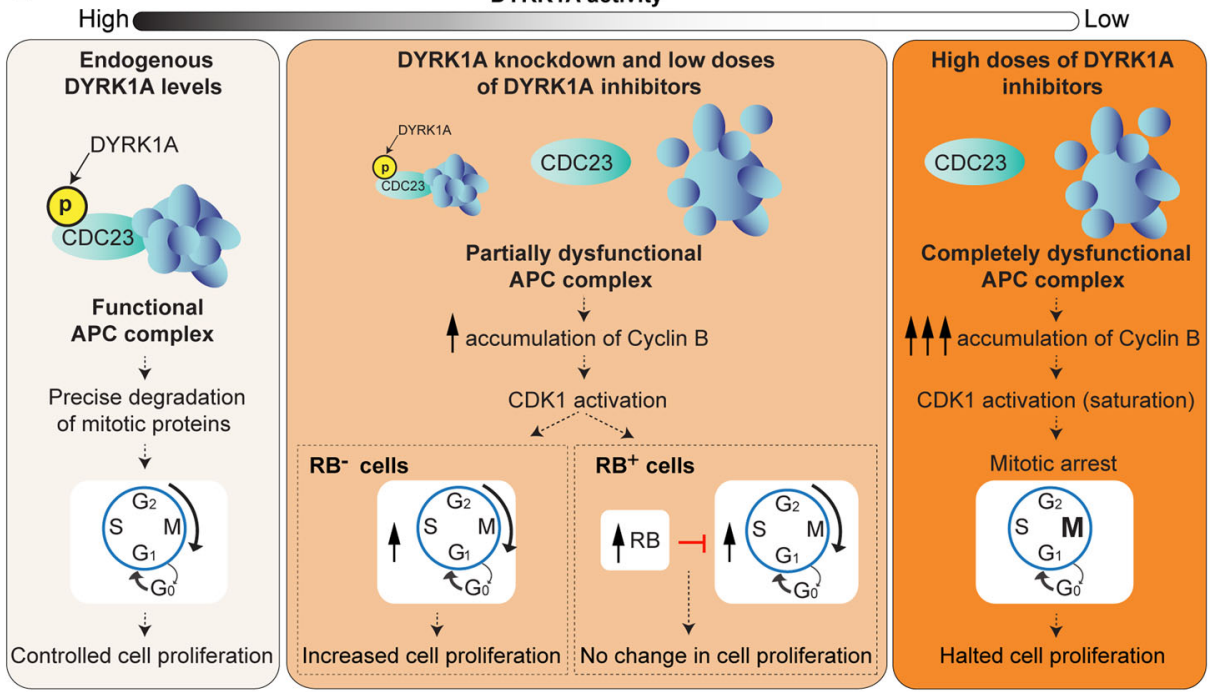

In summary, we delineated a novel DYRK1A-CDK1 signalling pathway. Importantly, we have also established that the phenotypic response to DYRK1A inhibition depends on both $\mathrm{RB}$ status and the degree of residual DYRK1A activity (Fig. 7L). Moderate DYRK1A inhibition is required to enhance proliferation in RB-deficient cells. 
(see figure on previous page)

Fig. 7 DYRK1A inhibition reactivates the cell cycle in dormant RB-deficient cells. A Schematic overview of the experimental design. Dormant MMK1 cells were isolated by treating parental MMK1 cell population with $0.5 \mu \mathrm{M}$ of colchicine (Colch) for 14 days. Dormant cells were then cultured in drug-free medium (drug holidays, DH) or treated with DYRK1A inhibitor ALGERNON (ALG). Scale bar: $10 \mu \mathrm{m}$. B Immunoblot analysis of DYRK1A (DYR) and retinoblastoma (RB) expression in parental and dormant MMK1 cells. Representative images of two independent experiments are shown. C, D RT-PCR analysis of cell cycle and dormant genes in dormant MMK1 cells. E Immunofluorescence images and quantification of Ki67-positive MMK1 cells. Dormant cells were treated \pm ALG $(1 \mu \mathrm{M}, 7$ days), then stained to detect Ki67 (pink), tubulin (green) and DAPI (blue). Scale bar: $5 \mu \mathrm{m}$. $\mathbf{F}$ Nuclear staining quantification of dormant MMK1 cells treated \pm ALG $(1 \mu M)$ for 7 days. G RT-PCR analysis of cell cycle genes in MMK1 cells treated as outlined in panel (E). $\mathbf{H}$ Immunoblot analysis and densitometric quantification of cyclin B (Cyc B) expression in dormant MMK1 cells treated \pm ALG $(1 \mu \mathrm{M})$ for $72 \mathrm{~h}$. I, J Images and nuclear staining quantification of cells treated with Colch \pm ALG $(1 \mu \mathrm{M})$ for 14 days. Scale bar: $10 \mu \mathrm{m}$. $\mathbf{K}$ Images and nuclear staining quantification of HW1 cells treated with Colch \pm ALG $(1 \mu \mathrm{M})$ for 14 days. Prior to drug treatments, cells were transfected with scramble (si Ctr) and RB (si RB) targeting siRNA (24 h). Scale bar: $10 \mu \mathrm{m}$. L Summary of the proposed role of DYRK1A in glioblastoma stem cell proliferation. Bar graphs represent mean \pm SEM from at least three independent experiments (two-tailed unpaired $t$-test; ${ }^{*} P<0.05$, ${ }^{* *} P<0.01$, $\left.{ }^{* * *} P<0.001,{ }^{* * * *} P<0.0001\right)$.

In contrast, complete DYRK1A inhibition results in the saturation of CDK1 activity and cell cycle arrest, regardless of RB status. These findings provide new insights into the complexity of DYRK1A signalling in cancer cells.

\section{Material and methods Cell culture}

A172 and U251 cells were obtained from the European Collection of Cell Cultures (EACC, UK) in 2014 and authenticated in 2020 by Cell Bank Australia using short tandem profiling. Cells were cultured in DMEM supplemented with 10\% FBS and Antibiotic-Antimycotic solution (Life Technologies, Carlsbad, CA, USA) at $37^{\circ} \mathrm{C}$ and $5 \% \mathrm{CO}_{2}$. Patient-derived HW1, RN1, MMK1 and RKI1 glioblastoma stem cells $^{34}$ were cultured in KnockOut DMEM/F-12 supplemented with StemPro NSC SFM, 2 mM GlutaMAX-ICTS, 20 ng/mL EGF, 10 ng/mL FGF- $\beta$ and Antibiotic-Antimycotic solution (all Life Technologies) as adherent cells on flasks coated with MatriGel (Corning, NY, USA). The protocols were approved by the Human Ethics Committee of the Royal Brisbane \& Women's Hospital (RBWH 2004/161). Cell cultures were routinely tested for mycoplasma infection and culturing did not exceed 15 passages.

\section{Doxycycline-inducible DYRK1A knockdown}

Recombinant DNA Plasmid: sense 5'-cccGTTCGGC TTGCACCGTCATTTCtcgaGAAATGA CGGTGCAAGC CGAACtttttc-3'; antisense 5'-tcgagaaaaaaGTTCGGCTT GCACCGTCATTTC tcgaGAAATGACGGTGCAAGCCG AAC-3'. The DYRK1A inducible knockdown was developed using a lentiviral single vector system containing an H1tetO-shRNA cassette and the codon-optimized tetR linked to $\mathrm{EGFP}^{46}$. shRNA for DYRK1A was developed into plasmids via $E$. coli transformation. Vector packaging system containing the shRNA-DYRK1A $(10 \mu \mathrm{g} / \mu \mathrm{L})$ was transfected via calcium phosphate precipitation method $^{47}$ into HEK293T cells in the presence of $25 \mu \mathrm{M}$ chloroquine. Concentrated lentivirus was added to U251 cells in a complete 'Tet-free' media containing $4 \mu \mathrm{g} / \mathrm{mL}$ Polybrene ${ }^{\circledR}$ for 16h. Infected live cells were sorted for GFP-positivity by flow cytometry using a BD FACS Influx (purity $>95 \%$ ). Cells were maintained in $100 \mu \mathrm{g} / \mathrm{mL}$ normocin for 7 days and hairpin expression was induced via treatment with $10 \mu \mathrm{g} / \mathrm{mL}$ doxycycline.

\section{Synthesis and characterization of ALGERNON (ALG)}

ALG was synthesized as previously reported ${ }^{22}$ and tested in-house using a radioactive DYRK1A kinase assay ${ }^{17}$. Residual DYRK1A activity in the presence of newly synthesized ALGERNON $(1 \mu \mathrm{M})$ was $36.3 \pm 4 \%(n=3)$.

\section{Phosphoproteome sample preparation}

Cells were lysed in SDC buffer, heated $\left(5 \mathrm{~min}, 95^{\circ} \mathrm{C}\right)$, cooled on ice and sonicated. A $10 \mu \mathrm{L}$ aliquot was diluted 1:5 in $8 \mathrm{M}$ urea and protein concentration was determined with BCA assay. Then, $200 \mu \mathrm{g}$ of protein was diluted in SDC buffer, alkylated with $10 \mathrm{mM}$ Tris(2-carboxyethyl) phosphine $/ 40 \mathrm{mM} 2$-chloroacetamide $\mathrm{pH} 8\left(5 \mathrm{~min}, 45^{\circ} \mathrm{C}\right)$, digested with 1:100 Lys-C and Trypsin overnight at $37^{\circ} \mathrm{C}$ with agitation $(1500 \mathrm{rpm})$. Phosphopeptides were enriched using the EasyPhos workflow ${ }^{24}$. Eluted phosphopeptides were dried in a SpeedVac concentrator (Eppendorf) and resuspended in MS loading buffer $(0.3 \%$ TFA/ $2 \%$ acetonitrile) prior to LC-MS/MS measurement.

\section{MS-based DYRK1A kinase assay}

CDC23 (100 ng; Novus Biological, Centennial, CO, USA) was incubated with DYRK1A (30 ng, prepared in-house ${ }^{17}$ ) in $25 \mu \mathrm{L}$ reaction buffer $(50 \mathrm{mM}$ HEPES $\mathrm{pH} 7.5,1 \mathrm{mM}$ EGTA, $0.01 \%$ Tween $20,10 \mathrm{mM} \mathrm{MgCl}_{2}, 1 \mathrm{mM}$ dithiothreitol) and $100 \mu \mathrm{M}$ ATP $\left(90 \mathrm{~min}, 30^{\circ} \mathrm{C}\right)$. Reactions were terminated by a $10 \mathrm{~min}$ incubation at $70^{\circ} \mathrm{C}$. The protein was reduced and alkylated with $10 \mathrm{mM}$ TCEP / $40 \mathrm{mM}$ $\mathrm{CAA} \mathrm{pH} 8\left(5 \mathrm{~min}, 45^{\circ} \mathrm{C}\right)$. Urea (final $2 \mathrm{M}$ ) was added and proteins were digested with $5 \mathrm{ng}$ of Lys-C and Trypsin $\left(18 \mathrm{~h}, 30^{\circ} \mathrm{C}\right)$. Peptides were desalted on StageTips containing styrene divinylbenzene-reverse phase sulfonate, eluted, dried and resuspended in MS loading buffer $(0.3 \%$ $\mathrm{TFA} / 2 \%$ acetonitrile) prior to LC-MS/MS measurement. 


\section{LC-MS/MS measurements and data processing}

Peptides and phosphopeptides were loaded onto a $40 \mathrm{~cm}$ column $(75 \mu \mathrm{m}$ inner diameter $)$ fused silica packed with $1.9 \mu \mathrm{M}$ C18 ReproSil particles (Dr. Maisch $\mathrm{GmBH}$ ) and maintained at $60^{\circ} \mathrm{C}$. A Dionex U3000 RSLC Nano HPLC system (Thermo Fisher Scientific) was interfaced with a Q Exactive HF X benchtop Orbitrap mass spectrometer using a NanoSpray Flex ion source (Thermo Fisher Scientific). Peptides were separated with a binary buffer system of $0.1 \%(\mathrm{v} / \mathrm{v})$ formic acid (buffer A) and $80 \%(\mathrm{v} / \mathrm{v})$ acetonitrile / $0.1 \%(\mathrm{v} / \mathrm{v})$ formic acid (buffer B). For phosphoproteome and in vitro kinase assay analysis, peptides were eluted at $350 \mathrm{~nL} / \mathrm{min}$ and separated with a gradient of 3-19\% buffer B over $40 \mathrm{~min}$, followed by $19-41 \%$ buffer B over 20 min. Peptides were analysed with a full scan $(350-1400 \mathrm{~m} / z ; R=60,000$ at $200 \mathrm{~m} / z)$ at a target of $3 \mathrm{e} 6$ ions, followed by up to ten data-dependent MS2 scans using HCD (target 1e5 ions; max. IT $50 \mathrm{~ms}$; isolation window $1.6 \mathrm{~m} / z$; NCE 27\%; min. AGC target 2e4), detected in the Orbitrap mass analyser $(R=15,000$ at $200 \mathrm{~m} / z)$. Dynamic exclusion $(30 \mathrm{~s})$ and Apex trigger (2-4s) were switched on. For single-run proteome analysis, peptides were eluted at $300 \mathrm{~nL} / \mathrm{min}$, separated with a gradient of $5-30 \%$ buffer B over $2 \mathrm{~h}$ and analysed with a full scan $(350-1400 \mathrm{~m} / z ; R=60,000$ at $200 \mathrm{~m} / \mathrm{z}$ ) at a target of 3e6 ions, followed by up to 20 data-dependent MS2 scans using HCD (target 1e5 ions; max. IT $28 \mathrm{~ms}$; isolation window $1.4 \mathrm{~m} / \mathrm{z}$; NCE $27 \%$; min. AGC target 1e4), detected in the Orbitrap mass analyser $(R=15,000$ at $200 \mathrm{~m} / z)$. Dynamic exclusion $(30 \mathrm{~s})$ was switched on. Raw MS data were processed using MaxQuant $^{48}$ (v1.6.6.0 and v1.6.0.9 for the proteome/ phosphoproteome and in vitro kinase assays respectively), searching against the Human UniProt database (January, 2019), using default settings with the addition of 'Phospho(STY)' as a variable modification. The 'Match between runs' option was turned on for all analyses.

\section{Phosphoproteomics data analysis}

Data analysis was performed using Perseus ${ }^{49}$ and $R$ software environments. Phosphopeptide intensities were transformed $(\log 2)$, and significantly regulated phosphopeptides identified by ANOVA (adj. $p<0.05$ ) and Dunnett's post hoc test $(p<0.05$ Ctr vs DOX, untreated vs inhibitors). Enrichment of gene ontology annotation (GOBP, GOMF, GOCC, KEGG, Pfam) and enrichment of kinase-substrate motifs (PhosphoSitePlus $)^{50}$ was performed using a Fisher exact test with Benjamini-Hochberg used for truncation (FDR $<0.02$ ). Motif analysis was performed of phosphopeptides regulated in both treatments using IceLogo ${ }^{51}$. Kina$\mathrm{sePA}^{52}$ was used to infer kinase activity in treatments vs controls ${ }^{53}$.

\section{siRNA transfection}

Cells $\left(5 \times 10^{5}\right)$ were transfected with $5 \mathrm{nM}$ control, DYRK1A, RB1, RBL2, CDC23 siRNA and Lipofectamine RNA iMAX, according to manufacturer's instructions (Life Technologies).

\section{Clonogenic assay}

Cells (U251, $3 \times 10^{3}$; HW1 and MMK1, $6 \times 10^{3}$ ) were transfected with DYRK1A and control siRNAs, or treated with ALG for 14 days, fixed and stained with $1 \%$ Toluidine Blue (Sigma Aldrich). Colonies were counted using the ImageJ software (colony area plugging) and normalized to untreated controls.

\section{Antibodies}

Antibodies used were from Cell Signaling (Danvers, MA, USA) against DYRK1A, P107, P16, p-RB, RB, pCycD1, CycD1, CycB1, p-CDK1, Ki67, GAPDH, antirabbit HRP-linked, anti-mouse HRP-linked; from Abcam (Cambridge, UK) against CDK1, p-CDK1, CDC23, LIN52; and from Santa Cruz Biotechnology (Dallas, TX, USA) against LIN37, DYRK1A, LIN9, P130.

\section{Immunoblotting}

Protein concentrations were determined with Pierce BCA assay kit (ThermoFisher Scientific \#23225). Here, $20-40 \mu \mathrm{g}$ of total protein was resolved on $4-12 \%$ SDSPAGE gels and transferred onto PVDF membranes using iBlot 2 (all Life Technologies). Blocking with 5\% skim milk (RT, $1 \mathrm{~h}$ ) was followed by overnight incubation at $4{ }^{\circ} \mathrm{C}$ with primary antibodies in $5 \%$ BSA in TBS-T. Membranes were incubated with secondary antibodies in $1 \%$ skim milk in TBS-T (RT, $1 \mathrm{~h})$. Detection was performed with Immobilon Western HRP Substrate Luminol-Peroxidase reagent (Merck Millipore) and the ChemiDoc MP Imaging System (Bio-Rad, Hercules, CA, USA). Densitometry quantification was done with ImageLab software (Bio-Rad).

\section{Immunoprecipitation}

Cell pellets were homogenized in IP buffer and incubated with Protein A/G Sepharose (Life Technologies; $1 \mathrm{~h}$, $4^{\circ} \mathrm{C}$ ). The precleared supernatants containing $1 \mathrm{mg}$ of protein were incubated with P130 antibody overnight at $4{ }^{\circ} \mathrm{C}$, then with Protein A/G Sepharose $\left(3 \mathrm{~h}, 4^{\circ} \mathrm{C}\right)$. Beads were washed with IP buffer and resuspended in NuPAGE LDS Sample Buffer and NuPAGE Sample Reducing Agent (Life Technologies). Bound proteins eluted from the immune complexes were denatured $\left(5 \mathrm{~min}, 95^{\circ} \mathrm{C}\right)$ and analysed by immunoblotting.

\section{Cycloheximide chase assay}

Cells $\left(5.0 \times 10^{5}\right)$ were transfected with DYRK1A and control siRNAs, or treated with ALG for $72 \mathrm{~h}$, then 
treated with cycloheximide $(30 \mu \mathrm{g} / \mathrm{mL})$, lysed with RIPA buffer and analysed by immunoblotting.

\section{Flow cytometry}

HW1 and MMK1 cells $(1.0 \times 106)$ were transfected with DYRK1A and Ctr siRNA (72 h), fixed and stained with propidium iodide $(50 \mu \mathrm{g} / \mathrm{mL})$ in the presence of RNase A $(100 \mu \mathrm{g} / \mathrm{mL}$, both Sigma Aldrich) for $1 \mathrm{~h}$ at $37^{\circ} \mathrm{C}$. Samples were analysed using LSRFortessa X-20 cytometer running FACSDiVa v6 software (BD Biosciences, Franklin Lakes, NJ, USA) and FlowJo v10.3 software.

\section{Immunofluorescence}

Cells were fixed with ice-cold 4\% PFA (20 min, RT), blocked in 5\% normal goat serum/PBS $(20 \mathrm{~min})$ and incubated with anti- $\alpha$-tubulin (1:10) and Ki67 (1:400) antibodies. Secondary antibodies were Alexa488conjugated anti-mouse IgG and Alexa594-conjugated anti-rabbit IgG both (Life Technologies). Cell nuclei were counterstained using Prolong Gold mounting media with DAPI (Life Technologies). Images were acquired with a Zeiss Axio Scope.A1 microscope using ZEN 2 blue edition software (Zeiss). Images were processed using Fiji.

\section{Nuclear staining}

Cells were stained with NUCLEAR-ID Red DNA stain $\left(30 \mathrm{~min}, 37^{\circ} \mathrm{C}, 5 \% \mathrm{CO}_{2}\right.$ ), washed and mounted with Dako Fluorescence Mounting Medium (ENZO, Farmingdale, NY, USA). Images were acquired on a Zeiss upright fluorescence Axio Scope.A1 microscope and analysed using ImageJ. In each replicate, at least nine randomly chosen images were taken and number of nuclei quantified after setting the threshold automatically, using Li filter and 'Analyse particle' function in Batch mode.

\section{RT-PCR}

Total RNA from cells was extracted using RNeasy mini kit (Qiagen, Hilden, Germany), retrotranscribed and amplified using Applied Biosystems High-Capacity cDNA Reverse Transcription kit (Life Technologies) as per manufacturer's instructions. RT-PCR was performed using KAPA SYBR FAST Universal 2X qPCR Master Mix and Qiagen QuantiTect Primer Assays using standard procedures in a LightCycler 480 . Threshold cycles $(\mathrm{Ct})$ were calculated using the LightCycler ${ }^{\circledR} 480$ software (all Roche, Basel, Switzerland). Relative quantification using the comparative $\mathrm{Ct}$ method was used to analyse the data output. Values were expressed as fold change over corresponding values for the control by the $2-\Delta \Delta \mathrm{Ct}$ method. Primers from QIAGEN: GAPDH, CDKN1A, ORC1, MCM5, NEK2, BUB1, N2RF1. Customized primers
(Integrated DNA Technologies, Coralville, IO, IA): $p 27$ (fwd: 5'-CTG ATG CTG TTG CTC GGT TA-3'; rv: 5'TGC AGA CTC TGG GAC ATC TG-3'), DEC (fwd: $5^{\prime}-$ GGT TAG CGG AGC AAT GCG CA-3'; rv: $5^{\prime}$-AAC CGG CAT TTG GGG AAC CGT C-3').

\section{Statistical analysis}

All assays were performed in at least three independent experiments, values are expressed as mean \pm SEM. Statistical comparisons were performed with GraphPad Prism v7 software, the null hypothesis was rejected at the 0.05 level. No statistical methods were used to predetermine sample size but our sample sizes are equivalent to those reported in previous similar publications.

\section{Acknowledgements}

We would like to thank Jeff Holst for help with lentiviral infections.

\section{Author details}

${ }^{1}$ Charles Perkins Centre and School of Medical Sciences, Faculty of Medicine and Health, The University of Sydney, Camperdown, NSW 2006, Australia.

${ }^{2}$ Charles Perkins Centre and School of Life and Environmental Sciences, Faculty of Science, The University of Sydney, Camperdown, NSW 2006, Australia. ${ }^{3}$ School of Chemistry, Faculty of Science, The University of Sydney, Camperdown, NSW 2006, Australia. ${ }^{4}$ Oncogenic Signalling Laboratory, Telethon Kids Institute, Perth Children's Hospital, 15 Hospital Avenue, Nedlands, WA 6009, Australia. ${ }^{5}$ QIMR Berghofer Medical Research Institute, 300 Herston Road, Herston, QLD 4006, Australia. ${ }^{6}$ Charles Perkins Centre and School of Mathematics and Statistics, Faculty of Science, The University of Sydney, Sydney, NSW 2006, Australia. ${ }^{7}$ Computational Systems Biology Group, Children's Medical Research Institute, University of Sydney, Westmead, NSW 2145, Australia

\section{Funding}

This work was funded by NHMRC grant APP1106145, Cancer Institute NSW fellowship 15/CDF/1-07 (L.M.), Professor Tony Basten Fellowship (A.R.). P.Y. is supported by NHMRC Investigator grant APP1173469 and S.J.H. is supported by University of Sydney Postdoctoral Fellowship.

\section{Data availability}

RAW and MaxQuant processed proteomics and phosphoproteomics data have been deposited to the PRIDE ProteomeXchange repository (https://www.ebi. ac.uk/pride/login), with the accession PXD020441, username reviewer52279@ebi.ac.uk and password pu5hv07C. Processed data are provided in the Supplementary Tables.

\section{Ethics statement}

Patient tumour tissue collection for isolation of glioblastoma stem cell lines used in this study were approved by the Royal Brisbane Women's Hospital (RBHW 2004/161) and QIMR Berghofer Human Research Ethics Committees (HREC/17/QRBW/577 Novel Therapies for Brain Cancer).

\section{Conflict of interest}

The authors declare no competing interests.

\section{Publisher's note}

Springer Nature remains neutral with regard to jurisdictional claims in published maps and institutional affiliations.

Supplementary information The online version contains supplementary material available at https://doi.org/10.1038/s41420-021-00456-6. 
Received: 25 November 2020 Revised: 19 January 2021 Accepted: 3 February 2021

Published online: 16 April 2021

\section{References}

1. Abbassi, R., Johns, T. G., Kassiou, M. \& Munoz, L. DYRK1A in neurodegeneration and cancer: molecular basis and clinical implications. Pharm. Ther. 151, 87-98 (2015).

2. Arbones, M. L., Thomazeau, A., Nakano-Kobayashi, A., Hagiwara, M. \& Delabar, J. M. DYRK1A and cognition: a lifelong relationship. Pharm. Ther. 194, 199-221 (2019).

3. Kumar, K. et al. Novel selective thiadiazine DYRK1A inhibitor lead scaffold with human pancreatic beta-cell proliferation activity. Eur. J. Med. Chem. 157, 1005-1016 (2018)

4. Ackeifi, C. et al. Pharmacologic and genetic approaches define human pancreatic beta cell mitogenic targets of DYRK1A inhibitors. JCI Insight 5, e132594 (2020).

5. Abdolazimi, Y. et al. CC-401 promotes beta-cell replication via pleiotropic consequences of DYRK1A/B inhibition. Endocrinology 159, 3143-3157 (2018).

6. Kumar, K. et al. Development of kinase-selective, harmine-based DYRK1A inhibitors that induce pancreatic human beta-cell proliferation. J. Med. Chem. 61, 7687-7699 (2018)

7. Pozo, N. et al. Inhibition of DYRK1A destabilizes EGFR and reduces EGFRdependent glioblastoma growth. J. Clin. Invest. 123, 2475-2487 (2013).

8. Lee, S. B. et al. An ID2-dependent mechanism for VHL inactivation in cancer. Nature 529, 172-177 (2016).

9. Liu, Q. et al. Tumor suppressor DYRK1A effects on proliferation and chemoresistance of AML cells by downregulating c-Myc. PLoS ONE 9, e98853 (2014).

10. Kim, J. et al. Ablation of miR-10b suppresses oncogene-induced mammary tumorigenesis and metastasis and reactivates tumor-suppressive pathways. Cancer Res. 76, 6424-6435 (2016).

11. Luna, J. et al. DYRK1A modulates C-MET in pancreatic ductal adenocarcinoma to drive tumour growth. Gut 68, 1465-1476 (2019).

12. Radhakrishnan, A. et al. A dual specificity kinase, DYRK1A, as a potential therapeutic target for head and neck squamous cell carcinoma. Sci. Rep. $\mathbf{6}$, 36132 (2016)

13. Malinge, S. et al. Increased dosage of the chromosome 21 ortholog Dyrk1a promotes megakaryoblastic leukemia in a murine model of Down syndrome. J. Clin. Invest. 122, 948-962 (2012).

14. Giraud, F. et al. Synthesis, protein kinase inhibitory potencies, and in vitro antiproliferative activities of meridianin derivatives. J. Med. Chem. 54, 4474-4489 (2011).

15. Gourdain, S. et al. Development of DANDYs, new 3,5-diaryl-7-azaindoles demonstrating potent DYRK1A kinase inhibitory activity. J. Med. Chem. 56, 9569-9585 (2013).

16. Tazarki, H. et al. New pyrido[3,4-g]quinazoline derivatives as CLK1 and DYRK1A inhibitors: synthesis, biological evaluation and binding mode analysis. Eur. J. Med. Chem. 166, 304-317 (2019).

17. Zhou, Q. et al. Structural optimization and pharmacological evaluation of inhibitors targeting dual-specificity tyrosine phosphorylation-regulated kinases (DYRK) and CDC-like kinases (CLK) in glioblastoma. J. Med. Chem. 60, 2052-2070 (2017)

18. Munoz, L. Non-kinase targets of protein kinase inhibitors. Nat. Rev. Drug Discov. 16, 424-440 (2017).

19. Gimple, R. C., Bhargava, S., Dixit, D. \& Rich, J. N. Glioblastoma stem cells: lessons from the tumor hierarchy in a lethal cancer. Genes Dev. 33, 591-609 (2019).

20. Litovchick, L., Florens, L. A., Swanson, S. K., Washburn, M. P. \& DeCaprio, J. A. DYRK1A protein kinase promotes quiescence and senescence through DREAM complex assembly. Genes Dev. 25, 801-813 (2011).

21. Debdab, M. et al. Leucettines, a class of potent inhibitors of cdc2-like kinases and dual specificity, tyrosine phosphorylation regulated kinases derived from the marine sponge Leucettamine B: modulation of alternative pre-RNA splicing. J. Med. Chem. 54, 4172-4186 (2011).

22. Nakano-Kobayashi, A. et al. Prenatal neurogenesis induction therapy normalizes brain structure and function in Down syndrome mice. Proc. Natl Acad. Sci. USA 114, 10268-10273 (2017).

23. Soppa, U. et al. The Down syndrome-related protein kinase DYRK1A phosphorylates p27(Kip1) and Cyclin D1 and induces cell cycle exit and neuronal differentiation. Cell Cycle 13, 2084-2100 (2014).
24. Humphrey, S. J., Karayel, O., James, D. E. \& Mann, M. High-throughput and high-sensitivity phosphoproteomics with the EasyPhos platform. Nat. Protoc. 13, 1897-1916 (2018).

25. Humphrey, S. J., Azimifar, S. B. \& Mann, M. High-throughput phosphoproteomics reveals in vivo insulin signaling dynamics. Nat. Biotechnol. 33, 990-995 (2015).

26. Soundararajan, M. et al. Structures of Down syndrome kinases, DYRKs, reveal mechanisms of kinase activation and substrate recognition. Structure 21, 986-996 (2013).

27. Deibler, R. W. \& Kirschner, M. W. Quantitative reconstitution of mitotic CDK1 activation in somatic cell extracts. Mol. Cell 37, 753-767 (2010).

28. Lindqvist, A., van Zon, W., Karlsson Rosenthal, C. \& Wolthuis, R. M. Cyclin B1Cdk1 activation continues after centrosome separation to control mitotic progression. PLoS Biol. 5, e123 (2007).

29. Lindqvist, A., Rodriguez-Bravo, V. \& Medema, R. H. The decision to enter mitosis: feedback and redundancy in the mitotic entry network. J. Cell Biol. 185, 193-202 (2009).

30. Pines, J. Cubism and the cell cycle: the many faces of the APC/C. Nat. Rev. Mol. Cell Biol. 12, 427-438 (2011).

31. Chang, L., Zhang, Z., Yang, J., McLaughlin, S. H. \& Barford, D. Atomic structure of the APC/C and its mechanism of protein ubiquitination. Nature $\mathbf{5 2 2}$, 450-454 (2015).

32. Terrano, D. T., Upreti, M. \& Chambers, T. C. Cyclin-dependent kinase 1mediated $\mathrm{BCl}-\mathrm{xL} / \mathrm{BCl}-2$ phosphorylation acts as a functional link coupling mitotic arrest and apoptosis. Mol. Cell Biol. 30, 640-656 (2010).

33. Eichhorn, J. M., Kothari, A. \& Chambers, T. C. Cyclin B1 overexpression induces cell death independent of mitotic arrest. PLOS ONE 9, e113283 (2014).

34. Stringer, B. W. et al. A reference collection of patient-derived cell line and xenograft models of proneural, classical and mesenchymal glioblastoma. Sci. Rep. 9, 4902 (2019).

35. MacDonald, J. et al. A systematic analysis of negative growth control implicates the DREAM complex in cancer cell dormancy. Mol. Cancer Res. 15, 371-381 (2017).

36. Recasens, A. \& Munoz, L. Targeting cancer cell dormancy. Trends Pharm. Sci. 40, 128-141 (2019).

37. Abbassi, R. H. et al. Lower tubulin expression in glioblastoma stem cells attenuates efficacy of microtubule-targeting agents. ACS Pharm. Transl. Sci. 2, 402-413 (2019).

38. An, E. \& Brognard, J. Orange is the new black: kinases are the new master regulators of tumor suppression. IUBMB Life 71, 738-748 (2019).

39. Baffi, T. R., Van, A. N., Zhao, W., Mills, G. B. \& Newton, A. C. Protein kinase C quality control by phosphatase PHLPP1 unveils loss-of-function mechanism in cancer. Mol. Cell 74, 378-392 (2019).

40. Qiao, R. et al. Mechanism of APC/CCDC20 activation by mitotic phosphorylation. Proc. Natl Acad. Sci. USA 113, E2570-E2578 (2016).

41. Wolf, F., Sigl, R. \& Geley, S. ' . . The end of the beginning': colk1 thresholds and exit from mitosis. Cell Cycle 6, 1408-1411 (2007).

42. Roewenstrunk, J. et al. A comprehensive proteomics-based interaction screen that links DYRK1A to RNF169 and to the DNA damage response. Sci. Rep. 9, 6014 (2019).

43. Menon, V. R. et al. DYRK1A regulates the recruitment of 53BP1 to the sites of DNA damage in part through interaction with RNF169. Cell Cycle 18, 531-551 (2019).

44. Dick, F. A. \& Rubin, S. M. Molecular mechanisms underlying RB protein function. Nat. Rev. Mol. Cell Biol. 14, 297-306 (2013).

45. Boichuk, S. et al. The DREAM complex mediates GIST cell quiescence and is a novel therapeutic target to enhance imatinib-induced apoptosis. Cancer Res. 73, 5120-5129 (2013)

46. Herold, M. J., van den Brandt, J., Seibler, J. \& Reichardt, H. M. Inducible and reversible gene silencing by stable integration of an shRNAencoding lentivirus in transgenic rats. Proc. Natl Acad. Sci. USA 105, 18507-18512 (2008).

47. Follenzi, A. \& Naldini, L. HIV-based vectors. Preparation and use. Methods Mol. Med. 69, 259-274 (2002)

48. Cox, J. \& Mann, M. MaxQuant enables high peptide identification rates, individualized p.p.b.-range mass accuracies and proteome-wide protein quantification. Nat. Biotechnol. 26, 1367-1372 (2008).

49. Tyanova, S. et al. The Perseus computational platform for comprehensive analysis of (prote)omics data. Nat. Methods 13, 731-740 (2016). 
50. Hornbeck, P. V. et al. PhosphoSitePlus, 2014: mutations, PTMs and recalibrations. Nucleic Acids Res. 43, D512-D520 (2015).

51. Colaert, N., Helsens, K., Martens, L., Vandekerckhove, J. \& Gevaert, K. Improved visualization of protein consensus sequences by icelogo. Nat. Methods $\mathbf{6}$, 786-787 (2009).
52. Yang, P. et al. KinasePA: phosphoproteomics data annotation using hypothesis driven kinase perturbation analysis. Proteomics $\mathbf{1 6}$ 1868-1871 (2016).

53. Yang, P. et al. Multi-omic profiling reveals dynamics of the phased progression of pluripotency. Cell Syst. 8, 427-445 (2019). 